\title{
The Relationship between Access to Mass Media and HIV/AIDS Related Knowledge, Beliefs and Behaviours in Kenya
}

\author{
Irene Muli, Stephen Lawoko* \\ Department of Public Health Sciences, Karolinska Institutet, Stockholm, Sweden \\ Email: ${ }^{\text {Stephen.lawoko@ki.se }}$
}

Received 9 February 2014; revised 5 March 2014; accepted 1 April 2014

Copyright $@ 2014$ by authors and Scientific Research Publishing Inc.

This work is licensed under the Creative Commons Attribution International License (CC BY).

http://creativecommons.org/licenses/by/4.0/

(c) (i) Open Access

\begin{abstract}
Aim: We scrutinized the association between access to mass media and HIV/AIDS related knowledge, beliefs and behaviours in Kenya. Methods: Data on a representative sample of Kenyan women between 15 - 30 years of age $(n=3909)$ was retrieved from the Kenyan demographic and health survey (DHS 2008) and analyzed using Chi-square tests and binary logistic regression. Results: Media use was common with over $70 \%$ of participants using radio at least once a week. Between $3 \%-30 \%$ of participants had poor to inadequate knowledge/beliefs about HIV/AIDS, with variations depending on demographic and social factors such as age, education, literacy, wealth and residential area. HIV/AIDS knowledge, beliefs and behaviours were associated with exposure to media, even after control for possible co-variation with social and demographic factor. Conclusion: Despite wide exposure to media among young Kenyan women, substantial proportions have poor to inadequate knowledge of the aetiology, risk/protective factors and control measures of HIV/AIDS. Yet, such knowledge was positively associated with media use. Media thus could ideally be used to implement a comprehensive awareness campaign in the general population about the aetiology, risk/protective factors and control measures in HIV/AIDS.
\end{abstract}

\section{Keywords}

HIV/AIDS, Behavior, Knowledge, Media, Women, Kenya

\section{Background}

Sexual and reproductive health constitutes one of the biggest health challenges among young women particu-

*Corresponding author.

How to cite this paper: Muli, I., \& Lawoko, S. (2014). The Relationship between Access to Mass Media and HIV/AIDS Related Knowledge, Beliefs and Behaviours in Kenya. Psychology, 5, 736-743. http://dx.doi.org/10.4236/psych.2014.57084 
larly in low-income settings. Though many of the millennium development goals (MDGs) are on target, relatively lower success has been reported with those MDG's related to sexual and reproductive health namely to improve maternal health, gender equality and combat HIV/AIDS through women's empowerment (UN, 2013). Unprotected sex remains the main cause among young people of ill sexual health especially in regions hit hardest by the HIV/AIDS epidemic. Discussing condom use and other safe sex practices remain taboo among young women in low-income (Malarcher, 2012; Nyamyeya, Sheriff, \& Kirya, 2007; Anne, Johnson, \& Cassel, 2005). High sexual partner change rates, early marriage, misconceptions about how HIV/AIDS is transmitted, protective factors, adherence to traditional cultural values against unprotected sex and poor utility of scarcely available reproductive health services continue to threaten the effective management of HIV/AIDS transmission among young people most particularly in Sub-Saharan Africa (Kyomuhendo, 2003; KDHS 2010; UBOS, 2011).

The near eradication and control of the HIV epidemic in high-income countries was established through behavioural changes achieved mostly through investments and national commitment to STI control programs, public education and antiretroviral therapy (Anne, Johnson, \& Cassel, 2005). In poor countries with constrained resources, poor educational infrastructure, and high illiteracy however, alternative channels to educate inhabitants on sexual and reproductive health are urgently needed. Media can play an important role in changing sexual behaviours, transforming negative beliefs and increasing knowledge (Anne, Johnson, \& Cassel, 2005; McQuail, 2010; Johnson, 2013; Bertrand \& Anhang, 2006; Bandura, 1977). The increasing availability of and access to information and communication technologies in low income countries (KDHS 2010; UBOS, 2011) thus hold promise for a new era in reproductive and sexual health management in poor countries.

Mass media theories concur that frequent use of media in behavioural presentations can influence perceptions, knowledge and attitudes as well as individual choices to change their behaviours. The social learning theory posits behavioural change through imitation of rewarded behaviour in mass media (Bandura, 1977). In communities where sex education is inaccessible due to poor infrastructural development but media use high (e.g. rural communities), the media can play an important role as a sex educator with a potential to lead to positive behavioural changes. In Sub-Saharan Africa where discussions about sex with authorities and elders is perceived as embarrassing and taboo among youth, young people have indicated the media as a top source of information regarding information about HIV/AIDS (Managing Media Monster, 2008).

In the current study, we scrutinize the association between access to mass media and HIV/AIDS related knowledge, beliefs and behaviours in young adult women in Kenya.

Kenya is characteristic of many Sub-Saharan African countries, with a largely young (70\% below 30 years of age), low educated population (over 50\% having no or incomplete primary education) and a literacy rate of about $85 \%$. Yet, almost all Kenyans are estimated to use radio as a source of information and news, more than half are television owners and more than $60 \%$ are mobile phone literate mostly using the text message service (KDHS, 2010). The potential of media, information and telecommunication technologies as a sexual and reproductive health educator thus is prominent.

The purpose of this study is to explore the association between access to media and HIV/AIDS related knowledge, beliefs and behaviours in Kenya.

More specifically the following research questions are addressed:

- Is there an association between media exposure and sexual behaviour among young-women age 15 - 30 in Kenya?

- Is there an association between exposure to media and reproductive health literacy (e.g. HIV/AIDS Knowledge/beliefs)?

In addressing these research questions, demographic and social covariates are considered in the analysis.

\section{Methodology}

\subsection{Demographic and Health Surveys}

The demographic and health surveys (DHS) are carried out in several developing countries and receive main funding from the United States Agency for International Development (USAID). The key objective of the DHS is to assist participating countries in monitoring their demographic and health situation on a five-year basis. The participating countries have the main responsibility for its implementation.

The survey procedure (e.g., organization and sampling methods) and instruments used have received ethical approval from the Institutional Review Board of Opinion Research Corporation (ORC) Macro International, In- 
corporated.

\subsection{Sampling in the Kenyan DHS}

The survey used a multi-stage stratified sampling design, to sample 3,465 men between 15 - 54 years and 8,444 women ranging between ages 15 - 49 years. For detailed procedure see the KDHS final report (KDHS, 2010). For this study, a sub-set of women aged 15 - 30 years that had engaged in sexual activity $(n=3909)$ was of interest.

\subsection{Variables}

At a broad level, the DHS provide detailed data on fertility, marriage, awareness/utility of family planning methods, nutritional status of women and children, awareness regarding sexually transmitted illnesses including HIV, maternal and child health, and mortality and individual/household demographic and social characteristics. For the current study, the following variables were of interest.

\subsubsection{Dependent Variables}

The reproductive health literacy indicators were the dependent variables in this study. Respondents were required to take a position (yes/no) on whether: i) they had used condom at last intercourse ii) not having sex reduces the risk for AIDS iii) using condoms reduces risk of AIDS iv) mosquito bites can transmit AIDS v) sharing food with person who has AIDS can transmit AIDS vi) a healthy person can have AIDS vii) AIDS can be acquired through witchcraft or supernatural means.

\subsubsection{Independent Variables}

The main independent variable for this study was exposure to media measured as use of radio, television or newspaper at least once a week, with response options yes/no for each media option.

\subsubsection{Covariates}

The demographic and social covariates considered were age, residential area, education, literacy, wealth, marital status, working status as these theoretically may co-vary with both knowledge and media access

\subsection{Statistical Analysis}

Data cleansing: Where deemed necessary, variable transformations were made to allow for meaningful statistical analysis (e.g. merging of categorical data to improve statistical power, but maintaining logical sub-groups). The SPSS statistical program was used in analysis. Chi-square tests were conducted in the bivariate assessment of associations between the independent and dependent factors. To control for potential co-variation, logistic regression modelling was used (i.e. controlling for demographic and social factors) (Nunez, Steyerberg, \& Nunez, 2011). Statistical significance was assumed at $p<0.05$.

\section{Results}

\subsection{Demographic and Social Characteristics of Respondents}

As indicated in Table 1, majority of the study sample were 25 - 30 years of age, resident in rural settings and with no/primary education. Relatively many participants were illiterate, belonged to poor households and were unemployed.

\subsection{Access to Media}

Many participants used radio (74.1\%), television (34.6\%) and newspapers (23\%) at least once a week.

\subsection{HIV/AIDS Behaviour, Knowledge and Beliefs by Demographic/Social Characteristics}

As shown in Table 1, the use of condoms in latest intercourse occurred least frequently among older participants in rural areas, with no/primary education, illiterates, most poverty stricken and never married participants. The belief that abstinence reduced risk for AIDS was lowest among younger, un-educated, illiterate and divorced 
Table 1. Associations between demographic/social variables and HIV/AIDS behaviour and knowledge/beliefs.

\begin{tabular}{|c|c|c|c|c|c|c|c|c|c|c|c|c|c|c|c|c|}
\hline \multirow[t]{2}{*}{$\begin{array}{l}\text { Demographic } \\
\text { variables }\end{array}$} & \multicolumn{2}{|c|}{$\begin{array}{l}\text { Didn't use } \\
\text { condom at last } \\
\text { intercourse }\end{array}$} & \multicolumn{2}{|c|}{$\begin{array}{l}\text { Reduced risk } \\
\text { of AIDS through } \\
\text { abstinence }\end{array}$} & \multicolumn{2}{|c|}{$\begin{array}{l}\text { Reduced chances } \\
\text { of AIDS by } \\
\text { condom use }\end{array}$} & \multicolumn{2}{|c|}{$\begin{array}{l}\text { Reduced chances } \\
\text { of AIDS by one } \\
\text { sex partner }\end{array}$} & \multicolumn{2}{|c|}{$\begin{array}{c}\text { Healthy } \\
\text { person can } \\
\text { have AIDS }\end{array}$} & \multicolumn{2}{|c|}{$\begin{array}{l}\text { Can get AIDS } \\
\text { from mosquito } \\
\text { bites }\end{array}$} & \multicolumn{2}{|c|}{$\begin{array}{l}\text { Can get } \\
\text { AIDS from } \\
\text { witchcraft }\end{array}$} & \multicolumn{2}{|c|}{$\begin{array}{l}\text { Can get Aids } \\
\text { from sharing } \\
\text { food }\end{array}$} \\
\hline & $\mathrm{n}$ & $\%$ of $n$ & $\mathrm{n}$ & $\%$ of $n$ & $\mathrm{n}$ & $\%$ of $n$ & $\mathrm{n}$ & $\%$ of $n$ & $\mathrm{n}$ & $\%$ of $n$ & $\mathrm{n}$ & $\%$ of $n$ & $\mathrm{n}$ & $\%$ of $n$ & $n$ & $\%$ of $n$ \\
\hline \multicolumn{17}{|l|}{ Age } \\
\hline $15-19$ & 496 & $78.8^{* * *}$ & 630 & $87.3^{*}$ & 580 & 81.9 & 621 & 92.8 & 623 & $89.6^{*}$ & 585 & 20.9 & 619 & 7.1 & 607 & 8.6 \\
\hline $20-24$ & 1329 & 87.7 & 1489 & 90.7 & 1378 & 83.7 & 1480 & 92.9 & 1464 & 92.9 & 1337 & 20.3 & 1449 & 6.6 & 1428 & 10.4 \\
\hline $25-30$ & 1518 & 92.8 & 1695 & 89.0 & 1549 & 84.8 & 1689 & 93.0 & 1675 & 91.0 & 1541 & 19.7 & 1670 & 6.2 & 1646 & 11.2 \\
\hline \multicolumn{17}{|l|}{ Residential } \\
\hline Urban & 1182 & $83.6^{* * *}$ & 1343 & 88.2 & 1282 & $86.8^{* * *}$ & 1337 & $94.6^{* *}$ & 1336 & $94.8^{* * *}$ & 1240 & $13.2^{* * *}$ & 1316 & $5.4^{*}$ & 1325 & $6.7 * * *$ \\
\hline Rural & 2221 & 91.5 & 2471 & 90.0 & 2226 & 82.2 & 2453 & 92.0 & 2426 & 89.7 & 2217 & 24.0 & 2422 & 7.1 & 2356 & 12.5 \\
\hline \multicolumn{17}{|l|}{ Education } \\
\hline Non & 518 & $98.8^{* * *}$ & 506 & $85.0 * *$ & 334 & $60.8^{* * *}$ & 492 & $84.1^{* * *}$ & 478 & $70.1^{* * *}$ & 414 & $41.8^{* * *}$ & 482 & $14.1^{* * *}$ & * 446 & $30.5^{* * *}$ \\
\hline Primary & 1851 & 91.1 & 2076 & 89.5 & 1956 & 85.5 & 2064 & 93.2 & 2047 & 92.9 & 1862 & 21.7 & 2033 & 6.3 & 2004 & +9.3 \\
\hline Secondary & 748 & 83.6 & 909 & 90.8 & 895 & 86.5 & 911 & 95.8 & 912 & 97.4 & 874 & 12.4 & 901 & 3.8 & 907 & 5.4 \\
\hline Higher & 286 & 68.9 & 323 & 91.6 & 323 & 90.7 & 323 & 96.3 & 325 & 97.5 & 313 & 3.8 & 322 & 4.0 & 324 & 9.0 \\
\hline \multicolumn{17}{|l|}{ Literacy } \\
\hline Cannot read & 654 & $97.6^{* * *}$ & 662 & $86.3^{* * *}$ & 476 & $67.4^{* * *}$ & 645 & $85.1^{* * *}$ & 632 & $73.7 * * *$ & 543 & $39.8^{* * *}$ & 637 & $14.4^{* * *}$ & * 589 & $28.5^{* * *}$ \\
\hline $\begin{array}{l}\text { Can read } \\
\text { some parts }\end{array}$ & 385 & 93.2 & 428 & 85.3 & 407 & 83.0 & 423 & 90.8 & 418 & 91.9 & 374 & 22.5 & 418 & 6.5 & 413 & 8.7 \\
\hline Can read & 2347 & 85.5 & 2710 & 90.9 & 2611 & 87.1 & 2709 & 95.2 & 2699 & 95.6 & 2533 & 15.4 & 2671 & 4.6 & 2666 & 6.7 \\
\hline \multicolumn{17}{|l|}{ Wealth } \\
\hline Poorest & 730 & $96.4^{* * *}$ & 752 & 87.9 & 604 & $73.2 * * *$ & 751 & $88.1^{* * *}$ & 736 & $77.9 * * *$ & 661 & $34.3^{* * *}$ & 732 & $10.9 * * *$ & * 695 & $23.0^{* * *}$ \\
\hline Poorer & 486 & 88.7 & 543 & 90.1 & 498 & 83.1 & 532 & 91.4 & 530 & 93.2 & 485 & 19.8 & 537 & 6.1 & 513 & 9.2 \\
\hline Middle & 511 & 91.6 & 595 & 91.8 & 550 & 86.0 & 591 & 95.1 & 580 & 96.0 & 540 & 20.2 & 580 & 6.2 & 576 & 9.0 \\
\hline Richer & 603 & 88.6 & 698 & 90.1 & 661 & 85.2 & 691 & 93.3 & 692 & 93.4 & 631 & 20.9 & 687 & 5.4 & 680 & 7.5 \\
\hline Richest & 1073 & 82.4 & 1226 & 88.4 & 1195 & 87.9 & 1225 & 95.2 & 1224 & 95.8 & 1146 & 11.6 & 1202 & 4.7 & 1217 & 6.1 \\
\hline \multicolumn{17}{|l|}{ Marital } \\
\hline $\begin{array}{l}\text { Never been } \\
\text { married }\end{array}$ & 611 & $59.4^{* * *}$ & 953 & $91.1^{*}$ & 926 & $88.2^{* * *}$ & 953 & $94.3 *$ & 955 & $93.6^{* * *}$ & 907 & $15.0^{* * *}$ & 949 & 5.8 & 948 & $6.0^{* * *}$ \\
\hline Married & 2402 & 96.8 & 2374 & 88.4 & 2134 & 81.6 & 2356 & 92.3 & 2330 & 90.9 & 2128 & 21.7 & 2324 & 6.3 & 2272 & 11.8 \\
\hline $\begin{array}{l}\text { only living } \\
\text { together }\end{array}$ & 188 & 94.7 & 188 & 89.9 & 175 & 85.1 & 187 & 93.6 & 182 & 91.8 & 163 & 22.1 & 171 & 6.4 & 176 & 12.5 \\
\hline Widowed & 35 & 65.7 & 55 & 94.5 & 51 & 92.2 & 55 & 94.5 & 53 & 94.3 & 50 & 16.0 & 56 & 7.1 & 54 & 13.0 \\
\hline Divorced & 26 & 89.5 & 54 & 81.5 & 42 & 69.0 & 52 & 82.7 & 52 & 71.2 & 46 & 26.1 & 53 & 13.2 & 47 & 19.1 \\
\hline $\begin{array}{l}\text { Not living } \\
\text { together }\end{array}$ & 141 & 78.0 & 190 & 93.7 & 180 & 88.9 & 187 & 95.2 & 190 & 92.6 & 169 & 26.0 & 185 & 10.3 & 184 & 12.0 \\
\hline \multicolumn{17}{|l|}{ Working } \\
\hline No & 1642 & 89.2 & 1834 & 89.3 & 1636 & $81.0^{* * *}$ & 1827 & $91.9 *$ & 1819 & $89.4^{* * *}$ & 1665 & 21.1 & 1789 & 5.4 & 1766 & 11.3 \\
\hline Yes & 1752 & 88.4 & 1467 & 89.6 & 1861 & 86.5 & 1951 & 93.9 & 1931 & 93.5 & 1786 & 19.1 & 1936 & 7.5 & 1903 & 9.5 \\
\hline
\end{tabular}


participants. The belief that condom use reduced chances of AIDS was less common among rural, un-educated, illiterate, poorest, divorced and unemployed participants. The belief that having one sex partner reduced the risk of AIDS was most common among urban, higher educated, literate, richest, "not living together" and employed participants. No other contrast appearing in Table 1 reached statistical significance at the $p<0.05$ level.

As indicated in Table 1, the belief that healthy persons can have AIDS was lowest in younger, rural, uneducated, illiterate, poorest, divorced and unemployed participants. The belief that mosquito bites can transmit AIDS was lowest among rural, uneducated, illiterate, poorest, never married participants. That one can get AIDS from witchcraft was most commonly believed among rural, uneducated, illiterate and poorest participants. A similar trend was observed with regard to the belief that one can get AIDS from sharing food.

\subsection{HIV/AIDS Behaviour, Knowledge and Beliefs by Media Exposure}

As exhibited in Table 2, the use of condoms in latest intercourse was more pronounced among frequent users of radio, television and newspapers. The belief that abstinence, use of condoms and single partnership reduced risk of AIDS was most common among users/readers of radio and newspapers. In addition, television users were more prone that non-users to believe that use of condoms and single partnership reduced risk of AIDS.

As indicated in Table 3, radio, television and newspaper users were consistently more prone to believe that healthy persons can have AIDS and less prone to believe mosquitoes can transmit AIDS, AIDS can be got through witchcraft and sharing food.

Table 2. Associations between Media exposure and condom use and HIV/AIDS knowledge/beliefs.

\begin{tabular}{|c|c|c|c|c|c|c|c|c|}
\hline \multirow[t]{2}{*}{ Independent variables } & \multicolumn{2}{|c|}{$\begin{array}{l}\text { Didn't use condom } \\
\text { at last intercourse }\end{array}$} & \multicolumn{2}{|c|}{$\begin{array}{l}\text { Reduced risk of AIDS } \\
\text { by no sex }\end{array}$} & \multicolumn{2}{|c|}{$\begin{array}{l}\text { Reduced chances } \\
\text { of AIDS by condom use }\end{array}$} & \multicolumn{2}{|c|}{$\begin{array}{l}\text { Reduced chances of } \\
\text { AIDS by one sex partner }\end{array}$} \\
\hline & (n) & $\%$ of $n$ & (n) & $\%$ of $n$ & (n) & $\%$ of $n$ & (n) & $\%$ of $n$ \\
\hline \multicolumn{9}{|l|}{ Radio use } \\
\hline No/less than once/w & (896) & $94.4^{* * *}$ & (950) & $86.4^{* *}$ & (773) & $74.8 * * *$ & $(941)$ & $89.2^{* * *}$ \\
\hline Yes > once/w & $(2505)$ & 86.7 & (2861) & 90.4 & $(2732)$ & 86.5 & (2846) & 94.1 \\
\hline \multicolumn{9}{|l|}{ Newspaper use } \\
\hline No/less than once/w & $(2650)$ & $91.5^{* * *}$ & (2921) & $88.7^{*}$ & (2626) & $81.5^{* * *}$ & (2895) & $92.1^{* * *}$ \\
\hline Yes $>$ once/w & (750) & 78.9 & (890) & 91.7 & (879) & 91.0 & (892) & 95.6 \\
\hline \multicolumn{9}{|l|}{ Television use } \\
\hline No/less than once/w & $(2262)$ & $91.9 * * *$ & $(2474)$ & 88.8 & (2209) & $81.3^{* * *}$ & (2458) & $91.5^{* * *}$ \\
\hline Yes $>$ once/w & (1141) & 82.6 & (1340) & 90.4 & (1299) & 88.4 & (1332) & 95.6 \\
\hline
\end{tabular}

Table 3. Associations between Media exposure and HIV/AIDS knowledge/beliefs.

\begin{tabular}{|c|c|c|c|c|c|c|c|c|}
\hline \multirow[t]{2}{*}{ Independent variables } & \multicolumn{2}{|c|}{$\begin{array}{l}\text { Healthy person } \\
\text { can have AIDS }\end{array}$} & \multicolumn{2}{|c|}{$\begin{array}{c}\text { Can get AIDS from } \\
\text { mosquito bites }\end{array}$} & \multicolumn{2}{|c|}{$\begin{array}{l}\text { Can get AIDS } \\
\text { from witchcraft }\end{array}$} & \multicolumn{2}{|c|}{$\begin{array}{l}\text { Can get Aids from } \\
\text { sharing food }\end{array}$} \\
\hline & (n) & $\%$ of $n$ & (n) & $\%$ of $n$ & (n) & $\%$ of $n$ & (n) & $\%$ of $n$ \\
\hline \multicolumn{9}{|l|}{ Radio use } \\
\hline No/less than once/w & (923) & $80.5^{* * *}$ & (826) & $28.0 * * *$ & (930) & $9.1^{* * *}$ & (886) & $18.8^{* * *}$ \\
\hline Yes $>$ once/w & (2836) & 95.1 & (2634) & 17.7 & (2805) & 5.6 & (2792) & 7.7 \\
\hline \multicolumn{9}{|l|}{ Newspaper use } \\
\hline No/less than once/w & (2867) & $89.6^{* * *}$ & (2605) & $23.5^{* * *}$ & (2856) & $7.0^{*}$ & (2792) & $12.3^{* * *}$ \\
\hline Yes > once/w & (892) & 97.4 & (855) & 9.7 & (879) & 4.8 & (886) & 4.6 \\
\hline \multicolumn{9}{|l|}{ Television use } \\
\hline No/less than once/w & (2424) & $89.1^{* * *}$ & (2207) & $23.3 * * *$ & (2425) & $7.4 * *$ & (2347) & $12.7^{* * *}$ \\
\hline Yes $>$ once/w & (1338) & 95.8 & (1256) & 14.6 & (1313) & 4.9 & (1334) & 6.4 \\
\hline
\end{tabular}




\subsection{HIV/AIDS Behaviour, Knowledge and Beliefs by Media Exposure: Adjusting for Demographic and Social Covariate}

After adjusting for potential demographic and social covariates, radio use increased the likelihood of using condom in latest intercourse (Table 4), believing that condom use reduces chances of AIDS (Table 4) and believing that healthy persons can have AIDS (Table 5). Newspaper use increased the likelihood of believing that condom use reduces chances of AIDS (Table 4) but reduced the likelihood of believing that mosquito bites can transmit AIDS (Table 5). All other adjusted associations exhibited in Table 4, Table 5 were not statistically significant at $p<0.05$ as indicated by the confidence intervals which include 1 .

Table 4. Logistic regression demonstrating the association between media exposure condom use and HIV/AIDS knowledge/beliefs.

\begin{tabular}{|c|c|c|c|c|c|c|c|c|c|c|c|c|}
\hline \multirow[t]{2}{*}{$\begin{array}{l}\text { Independent } \\
\text { variables }\end{array}$} & \multicolumn{3}{|c|}{$\begin{array}{l}\text { Use condom at } \\
\text { last intercourse }\end{array}$} & \multicolumn{3}{|c|}{$\begin{array}{l}\text { Reduced chances } \\
\text { of AIDS by condom use }\end{array}$} & \multicolumn{3}{|c|}{$\begin{array}{l}\text { Reduced risk of } \\
\text { AIDS by no sex }\end{array}$} & \multicolumn{3}{|c|}{$\begin{array}{c}\text { Reduced chances of } \\
\text { AIDS by one sex partner }\end{array}$} \\
\hline & OR & $\begin{array}{l}\text { Crude } \\
\text { CI }\end{array}$ & $\begin{array}{l}\text { Adjusted } \\
\text { CI* }\end{array}$ & OR & $\begin{array}{l}\text { Crude } \\
\text { CI }\end{array}$ & $\begin{array}{l}\text { Adjusted } \\
\text { CI* }^{*}\end{array}$ & OR & $\begin{array}{l}\text { Crude } \\
\text { CI }\end{array}$ & $\begin{array}{l}\text { Adjusted } \\
\text { CI* }^{*}\end{array}$ & OR & $\begin{array}{l}\text { Crude } \\
\text { CI }\end{array}$ & $\begin{array}{l}\text { Adjusted } \\
\mathrm{CI}^{*}\end{array}$ \\
\hline \multicolumn{13}{|l|}{ Radio use } \\
\hline Yes & 1.00 & & & 1.00 & & & 1.00 & & & 1.00 & & \\
\hline No & 0.387 & $\begin{array}{c}0.284- \\
0.526\end{array}$ & $\begin{array}{c}0.440- \\
0.914\end{array}$ & 0.464 & $\begin{array}{c}0.382- \\
0.565\end{array}$ & $\begin{array}{c}0.612- \\
0.984\end{array}$ & 0.677 & $\begin{array}{l}0.541- \\
0.846\end{array}$ & $\begin{array}{c}0.588- \\
1.002\end{array}$ & 0.513 & $\begin{array}{c}0.396- \\
0.664\end{array}$ & $\begin{array}{c}0.647- \\
1.227\end{array}$ \\
\hline \multicolumn{13}{|l|}{ Newspaper use } \\
\hline Yes & 1.00 & & & 1.00 & & & 1.00 & & & 1.00 & & \\
\hline no & 0.346 & $\begin{array}{c}0.277- \\
0.432\end{array}$ & $\begin{array}{c}0.640- \\
1.199\end{array}$ & 0.436 & $\begin{array}{c}0.339- \\
0.560\end{array}$ & $\begin{array}{c}0.452- \\
0.813\end{array}$ & 0.710 & $\begin{array}{c}0.545- \\
0.924\end{array}$ & $\begin{array}{c}0.540- \\
1.022\end{array}$ & 0.530 & $\begin{array}{c}0.374- \\
0.750\end{array}$ & $\begin{array}{c}0.664- \\
1.546\end{array}$ \\
\hline \multicolumn{13}{|l|}{ Television use } \\
\hline Yes & 1.00 & & & 1.00 & & & 1.00 & & & 1.00 & & \\
\hline No & 0.417 & $\begin{array}{c}0.336- \\
0.517\end{array}$ & $\begin{array}{c}0.771- \\
1.455\end{array}$ & 0.570 & $\begin{array}{c}0.567- \\
0.697\end{array}$ & $\begin{array}{c}0.661- \\
1.103\end{array}$ & 0.848 & $\begin{array}{c}0.680- \\
1.058\end{array}$ & $\begin{array}{c}0.617 \text { - } \\
1.089\end{array}$ & 0.496 & $\begin{array}{c}0.369- \\
0.668\end{array}$ & $\begin{array}{c}0.523- \\
1.124\end{array}$ \\
\hline
\end{tabular}

*Adjusted for age, residential area, education, literacy, wealth index, marital status and work.

Table 5. Logistic regression demonstrating the association between media exposure and HIV/AIDS knowledge/beliefs.

\begin{tabular}{|c|c|c|c|c|c|c|c|c|c|c|c|c|}
\hline \multirow[t]{2}{*}{$\begin{array}{l}\text { Independent } \\
\text { variables }\end{array}$} & \multicolumn{3}{|c|}{$\begin{array}{c}\text { Healthy person can } \\
\text { have AIDS }\end{array}$} & \multicolumn{3}{|c|}{$\begin{array}{l}\text { Can get AIDS from } \\
\text { mosquito bites }\end{array}$} & \multicolumn{3}{|c|}{$\begin{array}{l}\text { Can get AIDS from } \\
\text { witchcraft }\end{array}$} & \multicolumn{3}{|c|}{$\begin{array}{l}\text { Can get Aids from } \\
\text { sharing food }\end{array}$} \\
\hline & OR & $\begin{array}{l}\text { Crude } \\
\text { CI }\end{array}$ & $\begin{array}{l}\text { Adjusted } \\
\text { CI* }^{*}\end{array}$ & OR & $\begin{array}{l}\text { Crude } \\
\text { CI }\end{array}$ & $\begin{array}{l}\text { Adjusted } \\
\text { CI* }^{*}\end{array}$ & OR & $\begin{array}{l}\text { Crude } \\
\text { CI }\end{array}$ & $\begin{array}{l}\text { Adjusted } \\
\text { CI* }^{*}\end{array}$ & OR & $\begin{array}{l}\text { Crude } \\
\text { CI }\end{array}$ & $\begin{array}{l}\text { Adjusted } \\
\mathrm{CI}^{*}\end{array}$ \\
\hline \multicolumn{13}{|l|}{ Radio use } \\
\hline No & 0.214 & $\begin{array}{c}0.169- \\
0.271\end{array}$ & $\begin{array}{c}0.411- \\
0.738\end{array}$ & 1.00 & & & 1.00 & & & 1.00 & & \\
\hline Yes & 1.00 & & & 0.552 & $\begin{array}{c}0.460- \\
0.662\end{array}$ & $\begin{array}{c}0.846- \\
1.342\end{array}$ & 0.593 & $\begin{array}{l}0.451- \\
0.781\end{array}$ & $\begin{array}{c}0.741- \\
1.464\end{array}$ & 0.361 & $\begin{array}{c}0.290- \\
0.449\end{array}$ & $\begin{array}{l}0.627- \\
1.098\end{array}$ \\
\hline \multicolumn{13}{|l|}{ Newspaper use } \\
\hline No & 0.229 & $\begin{array}{c}0.149- \\
0.352\end{array}$ & $\begin{array}{c}0.376- \\
1.036\end{array}$ & 1.00 & & & 1.00 & & & 1.00 & & \\
\hline Yes & 1.00 & & & 0.562 & $\begin{array}{c}0.467- \\
0.676\end{array}$ & $\begin{array}{c}0.958- \\
1.549\end{array}$ & 0.643 & $\begin{array}{c}0.479- \\
0.862\end{array}$ & $\begin{array}{c}0.687- \\
1.482\end{array}$ & 0.466 & $\begin{array}{c}0.363- \\
0.599\end{array}$ & $\begin{array}{c}0.749- \\
1.448\end{array}$ \\
\hline \multicolumn{13}{|l|}{ Television use } \\
\hline No & 0.357 & $\begin{array}{c}0.266- \\
0.481\end{array}$ & $\begin{array}{c}0.593- \\
1.288\end{array}$ & 1.00 & & & 1.00 & & & 1.00 & & \\
\hline Yes & 1.00 & & & 0.349 & $\begin{array}{c}0.274- \\
0.446\end{array}$ & $\begin{array}{c}0.560- \\
0.981\end{array}$ & 0.660 & $\begin{array}{c}0.474- \\
0.938\end{array}$ & $\begin{array}{c}0.787- \\
1.843\end{array}$ & 0.346 & $\begin{array}{c}0.248- \\
0.483\end{array}$ & $\begin{array}{c}0.492- \\
1.088\end{array}$ \\
\hline
\end{tabular}

*Adjusted for age, residential area, education, literacy, wealth index, marital status and work. 


\section{Discussion}

\subsection{Findings in Contexts of Previous Works and Implications}

The general aim of this study was to assess for relationship between media exposure and sexual behaviour/ HIV/AIDS knowledge/beliefs. We found Media use, particularly radio, to be common among young women in Kenya. Between 3\% - 30\% of participants had poor knowledge/beliefs about HIV/AIDS, with variations depending on demographic and social factors such as age, education, literacy, wealth and residential area, consistent with previous findings (Malarcher, 2010; Anne, Johnson, \& Cassel, 2005; WHO, 2011), and drumming for urgent interventions particularly among young, poor, low educated women in rural setting. That as many as one in three participants have poor to inadequate knowledge of or subscribe to distorted beliefs about HIV/AIDS is worrying and should trigger urgent intervention with a potential to improve knowledge and attitudinal change.

Consistent with earlier research, media use was positively associated with most measures of HIV/AIDS knowledge, beliefs and behaviours, even after control for possible confounding with social and demographic factor, reverberating previous work in other settings (Anne, Johnson, \& Cassel, 2005; Bertrand, \& Anhang, 2006; Bertrand, O’Reilly, Denison, Anhang, \& Sweat, 2006) about the positive effects of media in health management. Considering the high proportion of users in Kenya, the media could serve in a focussed, systematic and comprehensive awareness campaign in the general population about the aetiology, risk/protective factors and control measures in HIV/AIDS alongside other initiatives.

\subsection{Methodological Strengths and Weaknesses}

The DHS surveys have now been implemented in Kenya every 5 years since 1989, with improvements in data quality over time. The surveys are nationally representative and instruments have been repeatedly validated and used in several studies. Further, the availability of several demographic and social individual and household characteristics provide the opportunity to control for important covariates. A challenge however lies in the increasing probability of incomplete responses as number of variables increase. This could have implications on generalization of findings to the population if non-response is systematic. The data was also collected by various interviewers who had undergone training. However, we cannot rule out the possibility of differences in accuracy between interviewers (i.e. interviewer bias). Recall bias is also known to be prevalent in cross-sectional studies, particularly when events long back in time are in question. Another weakness with cross-sectional data is its inability to establish causality. For example, it is plausible that individuals with poor knowledge about HIV/AIDS have a preference to shut out media exposure and not as we hypothesize that media may have affected individual knowledge. Research of longitudinal nature is needed to ascertain casual links. Thus, we can only assume associations in this study and not causality. These problems notwithstanding, the findings are consistent with previous works.

\subsection{Further Research}

Although media use is high in Kenya, and its use found to be associated with HIV/AIDS knowledge and attitudes, it appears many young Kenyans still bare distorted views about the aetiology, risk factors and control measures for HIV/AIDS, raising concerns about the actual availability, content and intensity of any HIV/AIDS related programmes in Kenyan media. A critical analysis of media content is crucial to inform development of comprehensive educational interventions to address HIV/AIDS in Kenyan media. In addition, research of qualitative nature (e.g. deep interviews) with women may exhibit further factors that may influence their choice to use/not use media beyond those controlled for in this study.

\subsection{Conclusion}

In conclusion, substantial proportions of young Kenyan women (3\% - 30\%) have poor to inadequate knowledge of the aetiology, risk/protective factors and control measures of HIV/AIDS, despite considerable media exposure. Yet, such knowledge was positively associated with media use. Media thus may have a positive effect on behaviour and knowledge in relation to HIV/AIDS in Kenya and could ideally be used to implement a systematic and comprehensive awareness campaign in the general population about the aetiology, risk/protective factors and control measures in HIV/AIDS. The outreach of such campaigns however should cover the most women (i.e. 
young, poor, low educated women in rural settings as found in this study).

\section{Acknowledgments}

The data used in this study were made available through MEASURE DHS Archive. The data were originally collected by the ORC Macro, Calverton USA. We are most grateful to ORC Macro for availing us the dataset and to all parties involved in the collection of this data in Kenya.

\section{References}

Anne, M., Johnson, C. H. M., \& Cassel, J. A. (2005). Social Determinants, Sexual Behaviour and Sexual Health. In R. G. W., Michael Marmot (Ed), Social Determinants of Health (2nd ed., pp. 318-340). Oxford: Oxford University Press.

Bandura, A. (1977). Social Learning Theory. Eaglewood Cliffs, NJ: Prentice Hall.

Bertrand, J. T., \& Anhang, R. (2006). The Effectiveness of Mass Media in Changing HIV/AIDS-Related Behaviour among Young People in Developing Countries. World health Organization Tehcnical Report Series, 938, 205-241.

Bertrand, J. T., O’Reilly, K., Denison, J., Anhang, R., \& Sweat, M. (2006). Systematic Review of the Effectiveness of Mass Communication Programs to Change HIV/AIDS-Related Behaviors in Developing Countries. Health Education Research, 21, 567-597. http://dx.doi.org/10.1093/her/cyl036

Johnson, M. A. (2013). More than Pop Culture: Depictions of HIV in the Media and the Effect on Viewer's Perception of Risk. Journal of Homosexuality, 60, 1117-1142. http://dx.doi.org/10.1080/00918369.2013.776423

Kyomuhendo, G. B. (2003). Low Use of Rural Maternity Services in Uganda: Impact of Women’s Status, Traditional Beliefs and Limited Resources. Reproductive Health Matters, 11, 16-26. http://dx.doi.org/10.1016/S0968-8080(03)02176-1

Malarcher, S. (2010). World Health Organization. Social Determinants of Sexual and Reproductive Health: Informing Future Research and Programme Implementation. Geneva: World Health Organization, 157 pp.

(2008). Managing the Media Monster: The Influence of Media (From Television to Text Messages) on Teen Sexual Behavior and Attitudes. Washington, DC: National Campaignto Prevent Teen and Unplanned Pregnancy, 1-58671-071-0.

McQuail, D. (2010). McQuail’s Mass Commmunication Theory (6th ed). London: Sage.

Nunez, E., Steyerberg, E. W., \& Nunez, J. (2011). Regression Modeling Strategies. Revistaespanola de Cardiologia, 64, 501-507. http://dx.doi.org/10.1016/j.recesp.2011.01.019

Nyamyeya, P., Sheriff, I. D., \& Kirya, M. T. (2007). Reproductive and Sexual Health Rights in Kenya, Tanzania and Uganda: An Advocacy and Communication Approach. FEMNET.

Uganda Bureau of Statistics (UBOS) and Macro International Inc. 2010 (2011). Uganda Demographic and Health Survey 2011. Calverton, Maryland: UBOS and Macro International Inc.

United Nations (2013). We Can End Poverty 2015: Millennium Development Goals Report 2013. UN, New York.

World Health Organization (2011). HIV Prevention in Generalized Epidemics: Optimal Interventions for Global Fund Applications : Recommendations for a Public Health Approach-2011. Geneva: World Health Organization; iv, 51pp. 